\title{
An Approach to Hybrid Smoothing for Linear Continuous-Time Systems with Non-Gaussian Noises
}

\author{
Gou Nakura
}

E-mail: gg9925_fiesta@ybb.ne.jp

\begin{abstract}
In this paper we study hybrid estimation for linear continuous-time systems with noises not to be restricted to be Gaussian. It is assumed that modes of the systems are not directly accessible. We consider optimal estimation problems to find both estimated states of the systems and a candidate of the distributions of the modes over the finite time interval. We adopt most probable trajectory (MPT) approach. Q. Zhang (2000) has presented hybrid filtering algorithm, i.e., causal estimation, by MPT approach. We consider both filtering and smoothing problems in this paper. We also consider the cases that mode transitions follow Markovian jump processes and present nearly optimal estimation algorithms for limiting mode distributions. We can expect better estimation performance by taking into consideration noncausal information of observations. The hybrid smoother is realized by two filters approach.

Key Words: Hybrid systems; Noncausal estimation; Non-Gaussian noise; Two filters approach; Smoothing
\end{abstract}

\section{Introduction}

It is very important to consider simultaneous estimation of both system states and inaccesible modes for hybrid systems with unknown modes $([5,9])$. This estimation is called hybrid estimation. By the hybrid estimation we often want to know current mode at each time through information of observation. However there exist cases that we want to know distributions of modes on long run time interval rather than each estimate of the modes themselves at each time to graps global performance over long time intervals, for example, distributions of active modes in solar systems $([6,20])$, distributions of active agents on formation or consensus via hybrid systems representation and so on.

Much work has been done for smoothing theory for both of continuous- and discrete-time systems ([7, 10, $11,12,13,14,15,17,18,19,21,22]$ and so on). Various researchers have studied the smoothing problems by various approach, for example, maximum likelihood $\operatorname{approach}([11,14,18])$, projection $\operatorname{approach}([15])$ and so on. It is well known that smoothers (noncausal estimators) more effectively estimates the states than filters (causal estimators). It is well known that utilization of accumulated information of observation improves estimation performance. Nevertheless, on research of estimation for hybrid systems, little work has been done from the point of view of the noncausal information of observation, i.e., smoothing. In [11] Helmick et al. have presented a fixed-interval smoothing algorithm for discrete-time Markovian jump systems by maximum likelihood (ML) approach. However they have considered the case with fully accessible modes and their approach is based on approximate approach to probability density functions (PDFs). While it is significant that optimality is guaranteed for estimation algorithms, in [5] and [9] Costa et al. have presented LMMSE filters to estimate both system states and inaccesible modes for continuous- and discrete-time Markovian jump systems affected by wide sense white noises, but in these LMMSE filters theory the optimality of estimation isn't guaranteed in the meaning that these filters aren't always MMSE. To the best of the author's knowledge the optimal smoothing problems in the cases with inaccesible modes have not yet fully investigated.

In this paper we study hybrid estimation for linear continuous-time systems with non-Gaussian noises. The concerned systems are general hybrid systems given below which aren't restricted to Markovian jump systems and where added noises aren't restricted to be Gaussian. It is assumed that modes of the systems are not directly accessible throughout this paper. We consider optimal estimation problems to find both estimated states of the systems and a candidate of the distributions of the modes over the finite time interval. We adopt most probable trajectory (MPT) approach to guarantee the optimality of estimation methods. On this approach, given information of observation, we consider optimal control problems where we seek optimal control by which averaged noises energies are minimized for averaged systems. In [23] Zhang has presented hybrid filtering algorithm by MPT approach. We consider both filtering and smoothing problems in this paper. We can expect better estimation performance by taking into consideration noncausal information of observations. The hybrid smoother is realized by two filters approach $([10,13,19,21,22])$. We also consider cases that mode transitions follow Markovian jump prosesses and clarify relationship between estimators based on the Markovian mode transition probabilities and ones 
based on the limiting probabilities, i.e., we show near optimality of the limiting estimators. Finally we give numerical examples and verify that we can obtain better estimation performance by smoothing than filtering.

Notations: Throughout this paper the superscript"'" stands for the matrix transposition, $\|\cdot\|$ denotes the Euclidean vector norm and $\|v\|_{R}^{2}$ also denotes the weighted norm $v^{\prime} R v$. $O$ denotes the matrix with all zero components.

\section{Problem Formulation}

Let $(\Omega, \mathcal{F}, \mathcal{P})$ be a probability space and, on this space, we consider the following system with mode transitions and noises which aren't restricted to be Gaussian.

$$
\begin{gathered}
\dot{x}(t)=A(t, \theta(t)) x(t)+w(t, \theta(t)), \\
x(0)=x_{0}, \theta(0)=i_{0} \\
y(t)=H(t, \theta(t)) x(t)+v(t, \theta(t))
\end{gathered}
$$

where $x \in \mathbf{R}^{\mathbf{n}}$ is the state, $w \in \mathbf{R}^{\mathbf{n}}$ is the exogenous random noise, $v \in \mathbf{R}^{\mathbf{k}}$ is the measurement noise, and $y \in \mathbf{R}^{\mathbf{k}}$ is the measured output. $x_{0}$ is an unknown initial state and it is assumed that a distribution of initial modes $i_{0}$ is given. The noises $w(\cdot)$ and $v(\cdot)$ aren't restricted to be Gaussian.

We assume that all these matrices are of compatible dimensions.

Let $\mathcal{M}=\{1,2, \cdots, m\}$ denote the state space of $\theta(t)$. In this paper it is assumed that the probability distribution of $\theta(\cdot)$ is unknown, but among a finite number of candidate distributions. Let $r \in \mathcal{N}_{0}=\left\{1,2, \cdots, n_{0}\right\}$, and let $\mathcal{P}=\left\{\phi^{(1)}(\cdot), \cdots, \phi^{\left(n_{0}\right)}(\cdot)\right\}$ denote the set of such candidate distributions on $\mathcal{M}$, i.e., for $r \in \mathcal{N}_{0}$ and $t \in[0, T], \phi^{(r)}(t)=\left(\phi_{1}^{(r)}(t), \cdots, \phi_{m}^{(r)}(t)\right)$ with $\phi_{i}^{(r)}(t) \geq 0$ and $\sum_{i=1}^{m} \phi_{i}^{(r)}(t)=1$.

The fixed-interval smoothing problem we address in this paper for the system (1) is to find the MPT (most probable trajectory) estimate of $x(t), t \in[0, T]$, over the finite horizon $[0, T]$, using the information available on the known part of the observation $y(\cdot)$ for the given distributions of initial mode $i_{0}$ and initial state $x_{0}$. We define the following performance indices for $r \in \mathcal{N}_{0}$ and $t \in[0, T]:$

$$
\begin{aligned}
& J_{0 t}^{(r)}\left(x_{0}, w, v\right) \\
:= & \int_{0}^{t} \sum_{i=1}^{m} \phi_{i}^{(r)}(s)\left(w^{\prime}(s, i) M(s, i) w(s, i)\right. \\
& +v^{\prime}(s, i) N(s, i) v(s, i) d s \\
& \quad+\left(x_{0}-\hat{x}_{0}\right)^{\prime} D_{0}\left(x_{0}-\hat{x}(0)\right) \\
& J_{0 T}^{(r)}\left(x_{0}, w, v\right) \\
:= & \int_{0}^{T} \sum_{i=1}^{m} \phi_{i}^{(r)}(s)\left(w^{\prime}(s, i) M(s, i) w(s, i)\right.
\end{aligned}
$$

$$
\begin{gathered}
+v^{\prime}(s, i) N(s, i) v(s, i) d s \\
+\left(x_{0}-\hat{x}_{0}\right)^{\prime} D_{0}\left(x_{0}-\hat{x}(0)\right) \\
+\left(x(T)-\hat{x}_{T}\right)^{\prime} D_{T}\left(x(T)-\hat{x}_{T}\right)
\end{gathered}
$$

where $\hat{x}_{0}$ is an initial estimate of $x_{0}$ and $\hat{x}_{T}$ is a terminal estimate of $x(T) . M(t, i)>O, N(t, i) \geq O, D_{0}>O$ and $D_{T}>O$ are symmetric matrices which reflect the uncertainties on the estimate $\hat{x}(0)$, the noises $w(\cdot)$ and $v(\cdot)$. Thus these performance indices mean the energies of noises, initial and terminal estimates under some uncertainties averaged by the mode distributions for each $r \in \mathcal{N}_{0}$. We consider the optimization problems to decide $w(\cdot, i), v(\cdot, i)$ and $r \in \mathcal{N}_{0}$ minimizing $J_{0 t}^{(r)}$ and $J_{0 T}^{(r)}$ utilizing the known parts of the observed information $\mathcal{Y}_{T}=\{y(s) \mid 0 \leq s \leq T\}$.

Since the mode $\theta(t)$ at each time is inaccessible, we cannot directly design estimators for the system (1) including the unknown modes. Also, even if the modes are accessible, the computational complexity can exponentially increase if we directly design the estimators for the system (1) including $\theta(t)$ explicitely. Hence we introduce the system averaged through the mode distributions for each $r \in \mathcal{N}_{0}$.

For notational simplicity, we adopt the following notation.

$$
\bar{F}^{(r)}(t)=\sum_{i=1}^{m} \phi_{i}^{(r)}(t) F(t, i)
$$

for a matrix function $F(t, i)$ and $r \in \mathcal{N}_{0}$. Similarly ${\overline{F_{1} F_{2}}}^{(r)}(t)=\sum_{i=1}^{m} \phi_{i}^{(r)}(t) F_{1}(t, i) F_{2}(t, i)$ for matrix functions $F_{1}(t, i)$ and $F_{2}(t, i)$ and so on. Using these notations, we can shift the drift term in the system (1) to $\bar{A}^{(r)}(t)$ as follows:

$$
d x(t)=\bar{A}^{(r)}(t) x(t) d t+w(t) d t
$$

where

$$
\begin{aligned}
& w(t) \\
= & w^{(r)}(t)=\left(A(t, \theta(t))-\bar{A}^{(r)}(t)\right) x(t)+w(t, \theta(t)) .
\end{aligned}
$$

By replacing the system noise $w(t, i)$ by $\left(\bar{A}^{(r)}(t)-\right.$ $A(t, i)) x(t)+w(t)$ and the observation noise $v(t, i)$ by $y(t)-H(t, i) x(t)$ in the performance indices (2) and (3), we define

$$
\begin{aligned}
& \quad L^{(r)}(t, x, w, y) \\
& :=\sum_{i=1}^{m} \phi_{i}^{(r)}(t)\left(\left[\left(\bar{A}^{(r)}(t)-A(t, i)\right) x+w\right]^{\prime}\right. \\
& \quad \times M(t, i)\left[\left(\bar{A}^{(r)}(t)-A(t, i)\right) x+w\right] \\
& \left.+(y-H(t, i) x)^{\prime} N(t, i)(y-H(t, i) x)\right) .
\end{aligned}
$$

Then we can define the following performance indices:

$$
\begin{aligned}
& J_{f}^{(r)}(t, x, w(\cdot)) \\
:= & \int_{0}^{t} L^{(r)}(s, x(s), w(s), y(s)) d s+\Phi_{0}(x(0))
\end{aligned}
$$




$$
\begin{aligned}
& J_{b}^{(r)}(t, x, w(\cdot)) \\
&:= \int_{t}^{T} L^{(r)}(s, x(s), w(s), y(s)) d s+\Phi_{T}(x(T)) \\
& J_{s}^{(r)}(t, x, w(\cdot)):=J_{f}^{(r)}(t, x, w(\cdot))+J_{b}^{(r)}(t, x, w(\cdot))
\end{aligned}
$$

where $\Phi_{0}(x(\cdot))=\left(x(\cdot)-\hat{x}_{0}\right)^{\prime} D_{0}\left(x(\cdot)-\hat{x}_{0}\right)$ and $\Phi_{T}(x(\cdot))=\left(x(\cdot)-\hat{x}_{T}\right)^{\prime} D_{T}\left(x(\cdot)-\hat{x}_{T}\right)$.

We consider the optimal control problems to minimize $J_{f}^{(r)}$ and $J_{s}^{(r)}=J_{f}^{(r)}+J_{b}^{(r)}$ for the given parts of $\mathcal{Y}_{T}$. Let $V_{f}^{(r)}(t, x)$ and $V_{b}^{(r)}(t, x)$ be the value functions of these control problems as follows:

$$
\begin{aligned}
V_{f}^{(r)}(t, x) & :=\inf _{w(\cdot)} J_{f}^{(r)}(t, x, w(\cdot)) \\
V_{b}^{(r)}(t, x) & :=\inf _{w(\cdot)} J_{b}^{(r)}(t, x, w(\cdot)) \\
V_{s}^{(r)}(t, x) & :=V_{f}^{(r)}(t, x)+V_{b}^{(r)}(t, x) \\
w_{f}^{(r) *}(t) & :=\arg \min \left\{J_{f}^{(r)}(t, x, w(t)): w \in \mathbf{R}^{\mathbf{n}}\right\} \\
w_{b}^{(r) *}(t) & :=\arg \min \left\{J_{b}^{(r)}(t, x, w(t)): w \in \mathbf{R}^{\mathbf{n}}\right\} \\
w_{s}^{(r) *}(t) & :=\arg \min \left\{J_{s}^{(r)}(t, x, w(t)): w \in \mathbf{R}^{\mathbf{n}}\right\}
\end{aligned}
$$

Then define

$$
\begin{aligned}
& \hat{x}_{f}^{(r)}(t):=\arg \min \left\{V_{f}^{(r)}(t, x): x \in \mathbf{R}^{\mathbf{n}}\right\}, \\
& V_{f}^{(r)}(t):=V_{f}^{(r)}\left(t, \hat{x}_{f}^{(r)}(t)\right)
\end{aligned}
$$

and

$$
\hat{r}_{f}(t):=\arg \min \left\{V_{f}^{(r)}(t): r \in \mathcal{N}_{0}\right\} .
$$

Then the most probable distribution is $\phi^{\left(\hat{r}_{f}(t)\right)}(\cdot)$. Let $\hat{x}_{f}(t)=\hat{x}_{f}^{\left(\hat{r}_{f}(t)\right)}(t)$ and we have

$$
\begin{aligned}
& V_{f}^{\left(\hat{r}_{f}(t)\right)}\left(t, \hat{x}_{f}(t)\right) \leq V_{f}^{(r)}\left(t, \hat{x}_{f}^{(r)}(t)\right) \\
\leq & V_{f}^{(r)}(t, x)=J_{f}^{(r)}\left(t, x, w_{f}^{(r) *}(t)\right) \leq J_{f}^{(r)}(t, x, w(t)) .
\end{aligned}
$$

Also define

$$
\begin{aligned}
& \hat{x}_{s}^{(r)}(t):=\arg \min \left\{V_{s}^{(r)}(t, x): x \in \mathbf{R}^{\mathbf{n}}\right\}, \\
& V_{s}^{(r)}(t):=V_{s}^{(r)}\left(t, \hat{x}_{s}^{(r)}(t)\right)
\end{aligned}
$$

and

$$
\hat{r}_{s}(t):=\arg \min \left\{V_{s}^{(r)}(t): r \in \mathcal{N}_{0}\right\} .
$$

Then the most probable distribution is $\phi^{\left(\hat{r}_{s}(t)\right)}(\cdot)$. Let $\hat{x}_{s}(t)=\hat{x}_{s}^{\left(\hat{r}_{s}(t)\right)}(t)$ and we have

$$
\begin{aligned}
& V_{s}^{\left(\hat{r}_{s}(t)\right)}\left(t, \hat{x}_{s}(t)\right) \leq V_{s}^{(r)}\left(t, \hat{x}_{s}^{(r)}(t)\right) \\
\leq & V_{s}^{(r)}(t, x)=J_{s}^{(r)}\left(t, x, w_{s}^{(r) *}(t)\right) \leq J_{s}^{(r)}(t, x, w(t)) .
\end{aligned}
$$

Now we define the following optimal estimators in the sense of most probable trajectories (MPTs).
Definition 2.1 Given the matrices $M, N, D_{0}$ and $D_{T},\left(\hat{r}_{f}(t), \hat{x}_{f}(t)\right), t \geq 0$, is called an optimal filter (in the MPT sense) if it minimizes $V_{f}^{(r)}(t, x)$. $\left(\hat{r}_{s}(t), \hat{x}_{s}(t)\right), 0 \leq t \leq T$, is called an optimal smoother (in the MPT sense) if it minimizes $V_{s}^{(r)}(t, x)$.

Then we formulate the following optimal hybrid estimation problems for the performance indices (4) and (5).

The Optimal Hybrid Filtering Problem for Linear Continuous-Time Systems:

Find the pair $\left(\hat{r}_{f}(t), \hat{x}_{f}^{\left(\hat{r}_{f}(t)\right)}(t)\right), t \in[0, T]$ minimizing the performance index (4) based on the causal part $\mathcal{Y}_{t}=\{y(s) \mid 0 \leq s \leq t\}$ of the observed information $\mathcal{Y}_{T}$

The Optimal Hybrid Smoothing Problem for Linear Continuous-Time Systems:

Find the pair $\left(\hat{r}_{s}(t), \hat{x}_{s}^{\left(\hat{r}_{s}(t)\right)}(t)\right), t \in[0, T]$ minimizing the performance index $(4)+(5)$ based on the whole observed information $\mathcal{Y}_{T}$

\section{Hybrid Estimation Algorithms}

\subsection{Optimal Hybrid Filtering}

The Hamilton-Jacobi-Bellman (HJB) equations associated with the forward control problem to minimize $J_{f}^{(r)}$ with regard to $w(\cdot)$ are given as follows:

$$
\begin{aligned}
\frac{\partial V_{f}^{(r)}(t, x)}{\partial t}=\min _{w}\left\{L^{(r)}(t, x, w, y(t))\right. & \\
& \left.-\left(\bar{A}^{(r)}(t) x+w\right)^{\prime} \frac{\partial V_{f}^{(r)}(t, x)}{\partial x}\right\} \\
V_{f}^{(r)}(0, x)= & \Phi_{0}(x), r \in \mathcal{N}_{0}
\end{aligned}
$$

Then we obtain the following minimizing $w(\cdot)$.

$$
\begin{aligned}
w_{f}^{(r) *}(t, x) & \\
=\left(\bar{M}^{(r)}(t)\right)^{-1} & \left(\frac{1}{2} \frac{\partial V_{f}^{(r)}(t, x)}{\partial x}\right. \\
& \left.-\bar{M}^{(r)}(t) \bar{A}^{(r)}(t) x+\overline{M A}^{(r)}(t) x\right)
\end{aligned}
$$

Let

$$
V_{f}^{(r)}(t, x)=x^{\prime} K_{f}^{(r)}(t) x+2\left(p_{f}^{(r)}(t)\right)^{\prime} x+q_{f}^{(r)}(t)
$$

for some functions $K_{f}^{(r)}, p_{f}^{(r)}$ and $q_{f}^{(r)}$ with appropriate dimensions. For any given $t$, by letting $\partial V_{f}^{(r)} / \partial x=0$, we obtain

$$
K_{f}^{(r)}(t) x+p_{f}^{(r)}(t)=0 .
$$

Since it can be shown that the matrix $K_{f}^{(r)}(t)$ is positive-definite (see Lemma 2 in [23]), we obtain

$$
\hat{x}_{f}^{(r)}(t)=-\left(K_{f}^{(r)}(t)\right)^{-1} p_{f}^{(r)}(t)
$$


as the minimizer of $V^{(r)}(t, x)$.

Then we obtain the following matrix differential equations, forward filter equations and scalar equations with initial conditions:

$$
\begin{gathered}
\dot{K}_{f}^{(r)}(t) \\
=-K_{f}^{(r)}(t)\left(\bar{M}^{(r)}(t)\right)^{-1} K_{f}^{(r)}(t)+\bar{A}^{\prime} M A^{(r)}(t) \\
-\bar{A}^{\prime} \bar{M}^{(r)}(t)\left(\bar{M}^{(r)}(t)\right)^{-1} \overline{M A}^{(r)}(t)+\bar{H}^{\prime} N H^{(r)}(t) \\
-{\overline{A^{\prime} M}}^{(r)}(t)\left(\bar{M}^{(r)}(t)\right)^{-1} K_{f}^{(r)}(t) \\
-K_{f}^{(r)}(t)\left(\bar{M}^{(r)}(t)\right)^{-1} \overline{M A}^{(r)}(t), K_{f}^{(r)}(0)=D_{0} \\
\dot{\hat{x}}_{f}^{(r)}(t)=-\left(K_{f}^{(r)}(t)\right)^{-1}\left[{\overline{A^{\prime} M A}}^{(r)}(t)\right. \\
-\bar{A}^{\prime} \bar{M}^{(r)}(t)\left(\bar{M}^{(r)}(t)\right)^{-1} \overline{M A}^{(r)}(t) \\
\left.+\bar{H}^{\prime} N H^{(r)}(t)\right] \hat{x}_{f}^{(r)}(t) \\
+\left(\bar{M}^{(r)}(t)\right)^{-1} \overline{M A}^{(r)}(t) \hat{x}_{f}^{(r)}(t) \\
+\left(K_{f}^{(r)}(t)\right)^{-1} \bar{H}^{\prime} N^{(r)}(t) y(t), \hat{x}_{f}^{(r)}(0)=\hat{x}_{0} \\
\dot{q}_{f}^{(r)}(t) \quad\left(\hat{x}_{f}^{(r)}(t)\right)^{\prime} K_{f}^{(r)}(t)\left(\bar{M}^{(r)}(t)\right)^{-1} K_{f}^{(r)}(t) \hat{x}_{f}^{(r)}(t) \\
+y^{\prime}(t) \bar{N}^{(r)}(t) y(t), q_{f}^{(r)}(0)=\hat{x}_{0}^{\prime} D_{0} \hat{x}_{0}
\end{gathered}
$$

We also obtain

$$
V_{f}^{(r)}(t)=-\left(\hat{x}_{f}^{(r)}(t)\right)^{\prime} K_{f}^{(r)}(t) \hat{x}_{f}^{(r)}(t)+q_{f}^{(r)}(t) .
$$

Now we have the following filtering algorithm, which gives the solution of the Optimal Hybrid Filtering Problem for Linear Continuous-Time Systems.

*** Optimal hybrid filtering algorithm***

Step 1) Obtain $K_{f}^{(r)}(t), \hat{x}_{f}^{(r)}(t)$ and $q_{f}^{(r)}(t)$ for $r \in \mathcal{N}_{0}$ and $t \in[0, T]$ by solving (7), (8) and (9)with with initial conditions.

Step 2) Choose $\hat{r}_{f}(t)$ that minimizes

$$
V_{f}^{(r)}(t)=-\left(\hat{x}_{f}^{(r)}(t)\right)^{\prime} K_{f}^{(r)}(t) \hat{x}_{f}^{(r)}(t)+q_{f}^{(r)}(t) .
$$

Then the most probable distribution is $\phi^{\left(\hat{r}_{f}(t)\right)}(\cdot)$ and the optimal filter is given by

$$
\left(\hat{r}_{f}(t), \hat{x}_{f}(t)\right)=\left(\hat{r}_{f}(t), \hat{x}_{f}^{\left(\hat{r}_{f}(t)\right)}(t)\right) .
$$

\subsection{Optimal Hybrid Smoothing}

The Hamilton-Jacobi-Bellman (HJB) equations associated with the backward control problem to minimize $J_{b}^{(r)}$ with regard to $w(\cdot)$ are given as follows:

$$
\begin{aligned}
-\frac{\partial V_{b}^{(r)}(t, x)}{\partial t}=\min _{w} & \left\{L^{(r)}(t, x, w, y(t))\right. \\
\left.+\left(\bar{A}^{(r)}(t) x+w\right)^{\prime} \frac{\partial V_{b}^{(r)}(t, x)}{\partial x}\right\} & \\
V_{b}^{(r)}(T, x) & =\Phi_{T}(x), r \in \mathcal{N}_{0}
\end{aligned}
$$

Then we obtain the following minimizing $w(\cdot)$.

$$
\begin{aligned}
& w_{b}^{(r) *}(t, x) \\
&=-\left(\bar{M}^{(r)}(t)\right)^{-1}\left(\frac{1}{2} \frac{\partial V_{b}^{(r)}(t, x)}{\partial x}\right. \\
&\left.+\bar{M}^{(r)}(t) \bar{A}^{(r)}(t) x-\overline{M A}^{(r)}(t) x\right)
\end{aligned}
$$

Let

$$
V_{b}^{(r)}(t, x)=x^{\prime} K_{b}^{(r)}(t) x+2\left(p_{b}^{(r)}(t)\right)^{\prime} x+q_{b}^{(r)}(t)(10)
$$

for some functions $K_{b}^{(r)}, p_{b}^{(r)}$ and $q_{b}^{(r)}$ with appropriate dimensions. For any given $t$, by letting $\partial V_{b}^{(r)} / \partial x=0$, we obtain

$$
K_{b}^{(r)}(t) x+p_{b}^{(r)}(t)=0
$$

The following lemma holds.

Lemma 3.1 The matrix $K_{b}^{(r)}(t)$ is positive-definite.

(Proof) See the appendix. (Q.E.D.)

Therefore we obtain

$$
\hat{x}_{b}^{(r)}(t)=-\left(K_{b}^{(r)}(t)\right)^{-1} p_{b}^{(r)}(t)
$$

as the minimizer of $V_{b}^{(r)}(t, x)$.

Then we obtain the following matrix differential equations, backward filter equations and scalar equations with terminal conditions:

$$
\begin{aligned}
& \dot{K}_{b}^{(r)}(t) \\
= & K_{b}^{(r)}(t)\left(\bar{M}^{(r)}(t)\right)^{-1} K_{b}^{(r)}(t)-{\overline{A^{\prime} M A}}^{(r)}(t) \\
& +{\overline{A^{\prime} M}}^{(r)}(t)\left(\bar{M}^{(r)}(t)\right)^{-1} \overline{M A}^{(r)}(t)-\bar{H}^{\prime} N H^{(r)}(t) \\
& -{\overline{A^{\prime} M}}^{(r)}(t)\left(\bar{M}^{(r)}(t)\right)^{-1} K_{b}^{(r)}(t) \\
& -K_{b}^{(r)}(t)\left(\bar{M}^{(r)}(t)\right)^{-1} \overline{M A}^{(r)}(t), K_{b}^{(r)}(T)=D_{T}
\end{aligned}
$$

$$
\begin{aligned}
& \dot{\hat{x}}_{b}^{(r)}(t)=\left(K_{b}^{(r)}(t)\right)^{-1}\left[{\overline{A^{\prime} M A}}^{(r)}(t)\right. \\
&-{\overline{A^{\prime} M}}^{(r)}(t)\left(\bar{M}^{(r)}(t)\right)^{-1} \overline{M A}^{(r)}(t) \\
&\left.+{\overline{H^{\prime} N H}}^{(r)}(t)\right] \hat{x}_{b}^{(r)}(t) \\
&+\left(\bar{M}^{(r)}(t)\right)^{-1} \overline{M A}^{(r)}(t) \hat{x}_{b}^{(r)}(t) \\
&-\left(K_{b}^{(r)}(t)\right)^{-1}{\overline{H^{\prime} N}}^{(r)}(t) y(t), \hat{x}_{b}^{(r)}(T)=\hat{x}_{T}
\end{aligned}
$$

$$
\begin{aligned}
& \dot{q}_{b}^{(r)}(t) \\
& =\hat{x}_{b}^{(r)^{\prime}}(t) K_{b}^{(r)}(t)\left(\bar{M}^{(r)}(t)\right)^{-1} K_{b}^{(r)}(t) \hat{x}_{b}^{(r)}(t) \\
& -y^{\prime}(t) \bar{N}^{(r)}(t) y(t), q_{b}^{(r)}(T)=\hat{x}_{T}^{\prime} D_{T} \hat{x}_{T}
\end{aligned}
$$

We also obtain

$$
V_{b}^{(r)}(t)=-\left(\hat{x}_{b}^{(r)}(t)\right)^{\prime} K_{b}^{(r)}(t) \hat{x}_{b}^{(r)}(t)+q_{b}^{(r)}(t) .
$$


Using (6) and (10), we can express $V_{s}^{(r)}(t, x)$ as

$$
\begin{aligned}
& V_{s}^{(r)}(t, x)=x^{\prime}\left[K_{f}^{(r)}(t)+K_{b}^{(r)}(t)\right] x \\
&+2\left[p_{f}^{(r)}(t)+p_{b}^{(r)}(t)\right]^{\prime} x+q_{f}^{(r)}(t)+q_{b}^{(r)}(t)
\end{aligned}
$$

Let

$$
\partial V_{s}^{(r)} / \partial x=0
$$

and we obtain the following form.

$$
\hat{x}_{s}^{(r)}(t)=-\left[K_{f}^{(r)}(t)+K_{b}^{(r)}(t)\right]^{-1}\left(p_{f}^{(r)}(t)+p_{b}^{(r)}(t)\right)
$$

Since $p_{f}^{(r)}(t)=-K_{f}^{(r)}(t) \hat{x}_{f}^{(r)}(t)$ and $p_{b}^{(r)}(t)=$ $-K_{b}^{(r)}(t) \hat{x}_{b}^{(r)}(t)$, for each candidate $r$ of given distributions, we can obtain the following form of smoothed estimate at time $t$ by the forward and backward filtered estimates.

$$
\hat{x}_{s}^{(r)}(t)=K_{s}^{(r)}(t)\left[K_{f}^{(r)}(t) \hat{x}_{f}^{(r)}(t)+K_{b}^{(r)}(t) \hat{x}_{b}^{(r)}(t)\right]
$$

where $K_{s}^{(r)}(t)=\left[K_{f}^{(r)}(t)+K_{b}^{(r)}(t)\right]^{-1}$.

Now we have the following smoothing algorithm, which gives the solution of the Optimal Hybrid Smoothing Problem for Linear ContinuousTime Systems.

*** Optimal hybrid smoothing algorithm***

Step 1) Obtain $K_{b}^{(r)}(t), \hat{x}_{b}^{(r)}(t)$ and $q_{b}^{(r)}(t)$ for $r \in \mathcal{N}_{0}$ and $t \in[0, T]$ by solving (11), (12) and (13) with terminal conditions.

Step 2) Choose $\hat{r}_{s}(t)$ that minimizes

$$
V_{s}^{(r)}(t)=V_{f}^{(r)}(t)+V_{b}^{(r)}(t)
$$

where

$$
V_{b}^{(r)}(t)=-\hat{x}_{b}^{(r)^{\prime}}(t) K_{b}^{(r)}(t) \hat{x}_{b}^{(r)}(t)+q_{b}^{(r)}(t) .
$$

Then the most probable distribution is $\phi^{\left(\hat{r}_{s}(t)\right)}(\cdot)$ and the optimal smoother is given by

$$
\begin{aligned}
& \quad\left(\hat{r}_{s}(t), \hat{x}_{s}(t)\right)=\left(\hat{r}_{s}(t), \hat{x}_{s}^{\left(\hat{r}_{s}(t)\right)}(t)\right) \\
& =\left(\hat{r}_{s}(t), K_{s}^{\left(\hat{r}_{s}(t)\right)}(t)\left[K_{f}^{\left(\hat{r}_{s}(t)\right)}(t) \hat{x}_{f}^{\left(\hat{r}_{s}(t)\right)}(t)\right.\right. \\
& \left.\left.\quad+K_{b}^{\left(\hat{r}_{s}(t)\right)}(t) \hat{x}_{b}^{\left(\hat{r}_{s}(t)\right)}(t)\right]\right)
\end{aligned}
$$

where $K_{s}^{\left(\hat{r}_{s}(t)\right)}(t)=\left[K_{f}^{\left(\hat{r}_{s}(t)\right)}(t)+K_{b}^{\left(\hat{r}_{s}(t)\right)}(t)\right]^{-1}$.

\section{Markovian Mode Transitions and Limiting Estimation}

In this section it is assumed that the mode transitions follow some Markovian jump processes.

For each $r \in \mathcal{N}_{0}\left\{\theta^{(r)}(t)\right\}$ is a homogeneous Markov process with right continuous trajectories and taking values on the finite set $\mathcal{M}=\{1,2, \cdots, m\}$ with the following stationary transition probabilities:

$$
\begin{aligned}
\mathcal{P}\left\{\theta^{(r)}(t+\Delta)=j \mid \theta^{(r)}(t)=i\right\} & \left\{\begin{array}{cc}
\pi_{i j}^{(r)} \Delta+o(\Delta) & i \neq j \\
1+\pi_{i i}^{(r)} \Delta+o(\Delta) & i=j
\end{array}\right.
\end{aligned}
$$

where $\pi_{i j}^{(r)} \geq 0$ is the transition rate from the state $i$ to $j, i \neq j$, and $\pi_{i i}^{(r)}=-\sum_{j=1, j \neq i}^{N} \pi_{i j}^{(r)}<0$. Let $\Pi^{(r)}=$ $\left[\left(\pi_{i j}^{(r)}\right)\right]$ be the transition matrix of $\theta^{(r)}(t)$ and define $\phi_{i}^{(r)}(t):=\mathcal{P}\left\{\theta^{(r)}(t)=i\right\}>0$ for any $i \in \mathcal{M}([8,9])$.

Let $\phi^{(r)}(t)$ denote the probability distribution of $\theta(t)^{(r)}$. Then $\phi^{(r)}(t)$ satisfies the Kolmogorov forward differential equation

$$
\dot{\phi}^{(r)}(t)=\phi^{(r)}(t) \Pi^{(r)}(t)
$$

for the given initial distribution $\phi^{(r)}(0)([8,9])$.

Definition 4.1 For each $r \in \mathcal{N}_{0}$ and $t, \Pi^{(r)}(t)=$ $\left[\left(\pi_{i j}^{(r)}(t)\right)\right]$ is weakly irreducible if the equations

$$
\nu^{(r)}(t) \Pi^{(r)}(t)=0 \text { and } \sum_{i=1}^{m} \nu_{i}^{(r)}(t)=1
$$

have a unique nonnegative solution $\nu^{(r)}(t)=\left(\nu_{1}^{(r)}(t), \cdots, \nu_{m}^{(r)}(t)\right)$. Then the solution $\nu(t)$ is called the quasi-stationary distribution corresponding to $\Pi^{(r)}(t)$.

We consider the generator of the Markov chain with fast and slow motions as follows:

For sufficiently small $\epsilon>0$,

$$
\Pi^{(r), \epsilon}(t)=\frac{1}{\epsilon} \tilde{\Pi}^{(r)}(t)+\hat{\Pi}^{(r)}(t) .
$$

Let $\phi^{(r), \epsilon}(\cdot)$ denote the solution of (16) with $\Pi^{(r)}(t)$ replaced by $\Pi^{(r), \epsilon}(t)$.

For simplicity, we assume that $\tilde{\Pi}^{(r)}(t)$ consists of a single block of irreducible matrix, i.e., $\tilde{\Pi}^{(r)}(t)$ itself is weakly irreducible. Then define $\tilde{\phi}^{(r)}(t):=$ $\lim _{\epsilon \rightarrow 0} \tilde{\phi}_{i}^{(r), \epsilon}(t)$. Let $\tilde{\nu}^{(r)}(t)$ denote the quasi-stationary distribution corresponding to $\tilde{\Pi}^{(r)}(t)$ and $\tilde{\nu}^{(r)}(t)=$ $\tilde{\phi}^{(r)}(t)$ holds.

We adopt the notation

$$
\bar{F}_{\lambda}^{(r)}(t)=\sum_{i=1}^{m} \tilde{\phi}_{i}^{(r)}(t) F(t, i)
$$

for any matrix function $F(t, i)$ and $r \in \mathcal{N}_{0}$.

Then, for some $\tilde{K}_{f}^{(r)}(t), \quad \tilde{x}_{f}^{(r)}(t), \quad \tilde{q}_{f}^{(r)}(t), \tilde{K}_{b}^{(r)}(t)$, $\tilde{x}_{b}^{(r)}(t)$ and $\tilde{q}_{b}^{(r)}(t)$, consider the following differential equations with the initial or terminal conditions:

$$
\begin{aligned}
& \dot{\tilde{K}}_{f}^{(r)}(t) \\
= & -\tilde{K}_{f}^{(r)}(t)\left(\bar{M}_{\lambda}^{(r)}(t)\right)^{-1} \tilde{K}_{f}^{(r)}(t)+{\overline{A^{\prime} M A_{\lambda}}}^{(r)}(t) \\
& -{\overline{A^{\prime} M}}_{\lambda}^{(r)}(t)\left(\bar{M}_{\lambda}^{(r)}(t)\right)^{-1} \overline{M A}_{\lambda}^{(r)}(t)+\bar{H}^{\prime} N H_{\lambda}^{(r)}(t) \\
& -{\overline{A^{\prime} M}}_{\lambda}^{(r)}(t)\left(\bar{M}_{\lambda}^{(r)}(t)\right)^{-1} \tilde{K}_{f}^{(r)}(t) \\
& -\tilde{K}_{f}^{(r)}(t)\left(\bar{M}_{\lambda}^{(r)}(t)\right)^{-1} \overline{M A}_{\lambda}^{(r)}(t), \tilde{K}_{f}^{(r)}(0)=D_{0}
\end{aligned}
$$




$$
\begin{aligned}
& \dot{\tilde{x}}_{f}^{(r)}(t)=-\left(\tilde{K}_{f}^{(r)}(t)\right)^{-1}\left[{\overline{A^{\prime} M A_{\lambda}}}_{\lambda}^{(r)}(t)\right. \\
& -{\overline{A^{\prime} M_{\lambda}^{(r)}}}^{(t)}\left(\bar{M}_{\lambda}^{(r)}(t)\right)^{-1} \overline{M A}_{\lambda}^{(r)}(t) \\
& \left.+{\overline{H^{\prime} N H_{\lambda}}}_{\lambda}^{(r)}(t)\right] \tilde{x}_{f}^{(r)}(t) \\
& +\left(\bar{M}_{\lambda}^{(r)}(t)\right)^{-1} \overline{M A}_{\lambda}^{(r)}(t) \tilde{x}_{f}^{(r)}(t) \\
& +\left(\tilde{K}_{f}^{(r)}(t)\right)^{-1} \bar{H}^{\prime} N_{\lambda}^{(r)}(t) y(t), \tilde{x}_{f}^{(r)}(0)=\hat{x}_{0}
\end{aligned}
$$

$$
\begin{aligned}
& \dot{\tilde{q}}_{f}^{(r)}(t) \\
& =-\left(\tilde{x}_{f}^{(r)}(t)\right)^{\prime} \tilde{K}_{f}^{(r)}(t)\left(\bar{M}_{\lambda}^{(r)}(t)\right)^{-1} \tilde{K}_{f}^{(r)}(t) \tilde{x}_{f}^{(r)}(t) \\
& +y^{\prime}(t) \bar{N}_{\lambda}^{(r)}(t) y(t), \tilde{q}_{f}^{(r)}(0)=\hat{x}_{0}^{\prime} D_{0} \hat{x}_{0}
\end{aligned}
$$

$$
\begin{aligned}
& \dot{\tilde{K}}_{b}^{(r)}(t) \\
& =\tilde{K}_{b}^{(r)}(t)\left(\bar{M}_{\lambda}^{(r)}(t)\right)^{-1} \tilde{K}_{b}^{(r)}(t)-{\overline{A^{\prime} M A_{\lambda}^{(r)}}}^{(r)} \\
& +{\overline{A^{\prime} M}}_{\lambda}^{(r)}(t)\left(\bar{M}_{\lambda}^{(r)}(t)\right)^{-1} \overline{M A}_{\lambda}^{(r)}(t)-{\overline{H^{\prime} N H_{\lambda}}}^{(r)}(t) \\
& -{\overline{A^{\prime} M_{\lambda}}}_{\lambda}^{(r)}(t)\left(\bar{M}_{\lambda}^{(r)}(t)\right)^{-1} \tilde{K}_{b}^{(r)}(t) \\
& -\tilde{K}_{b}^{(r)}(t)\left(\bar{M}_{\lambda}^{(r)}(t)\right)^{-1} \overline{M A}_{\lambda}^{(r)}(t), \tilde{K}_{b}^{(r)}(T)=D_{T} \\
& \dot{\tilde{x}}_{b}^{(r)}(t)=\left(\tilde{K}_{b}^{(r)}(t)\right)^{-1}\left[{\overline{A^{\prime} M A}}_{\lambda}^{(r)}(t)\right. \\
& -{\overline{A^{\prime} M_{\lambda}^{(r)}}}^{(t)}\left(\bar{M}_{\lambda}^{(r)}(t)\right)^{-1} \overline{M A}_{\lambda}^{(r)}(t) \\
& \left.+{\overline{H^{\prime} N H_{\lambda}}}_{\lambda}^{(r)}(t)\right] \tilde{x}_{b}^{(r)}(t) \\
& +\left(\bar{M}_{\lambda}^{(r)}(t)\right)^{-1} \overline{M A}_{\lambda}^{(r)}(t) \tilde{x}_{b}^{(r)}(t) \\
& -\left(\tilde{K}_{b}^{(r)}(t)\right)^{-1}{\overline{H^{\prime} N_{\lambda}^{(r)}}}^{(t)} y(t), \tilde{x}_{b}^{(r)}(T)=\hat{x}_{T}
\end{aligned}
$$

$$
\begin{aligned}
& \quad \dot{\tilde{q}}_{b}^{(r)}(t) \\
& =\tilde{x}_{b}^{(r)^{\prime}}(t) \tilde{K}_{b}^{(r)}(t)\left(\bar{M}_{\lambda}^{(r)}(t)\right)^{-1} \tilde{K}_{b}^{(r)}(t) \tilde{x}_{b}^{(r)}(t) \\
& \quad-y^{\prime}(t) \bar{N}_{\lambda}^{(r)}(t) y(t), \tilde{q}_{b}^{(r)}(T)=\hat{x}_{T}^{\prime} D_{T} \hat{x}_{T}
\end{aligned}
$$

Then the following proposition holds.

Proposition 4.1 For $r \in \mathcal{N}_{0}$, let $K_{f}^{(r), \epsilon}(t), x_{f}^{(r), \epsilon}(t)$, $q_{f}^{(r), \epsilon}(t), K_{b}^{(r), \epsilon}(t), x_{b}^{(r), \epsilon}(t)$ and $q_{b}^{(r), \epsilon}(t)$ denote the solutions of the differential equations (7)-(9) and (11)-(13) respectively with $\phi^{(r)}(\cdot)$ replaced by $\phi^{(r), \epsilon}(\cdot)$. Then

$$
\begin{aligned}
K_{f}^{(r), \epsilon}(t) & \rightarrow \tilde{K}_{f}^{(r)}(t) K_{b}^{(r), \epsilon}(t) \rightarrow \tilde{K}_{b}^{(r)}(t) \\
\hat{x}_{f}^{(r), \epsilon}(t) & \rightarrow \tilde{x}_{f}^{(r)}(t) \hat{x}_{b}^{(r), \epsilon}(t) \rightarrow \tilde{x}_{b}^{(r)}(t) \\
q_{f}^{(r), \epsilon}(t) & \rightarrow \tilde{q}_{f}^{(r)}(t) q_{b}^{(r), \epsilon}(t) \rightarrow \tilde{q}_{b}^{(r)}(t)
\end{aligned}
$$

uniformly hold on $[0, T]$ as $\epsilon \rightarrow 0$ where $\tilde{K}_{f}^{(r)}(t), \tilde{x}_{f}^{(r)}(t)$, $\tilde{q}_{f}^{(r)}(t), \tilde{K}_{b}^{(r)}(t), \tilde{x}_{b}^{(r)}(t)$ and $\tilde{q}_{b}^{(r)}(t)$ are the unique solutions of (17)-(19) and (20)-(22) respectively.

(Proof) See the appendix. (Q.E.D.)

The limiting smoother $\left(\tilde{r}_{s}(t), \tilde{x}_{s}(t)\right)$ can be obtained as follows: Solve (17)-(19) with the initial conditions

and (20)-(22) with the terminal conditions and obtain $\tilde{K}_{f}^{(r)}(t), \tilde{x}_{f}^{(r)}(t), \tilde{q}_{f}(t), \tilde{K}_{b}^{(r)}(t), \tilde{x}_{b}^{(r)}(t)$ and $\tilde{q}_{b}(t), t \in$ $[0, T]$. Then, for each $t$, choose $\tilde{r}_{s}(t)$ that minimizes

$$
\tilde{V}_{s}^{(r)}(t)=\tilde{V}_{f}^{(r)}(t)+\tilde{V}_{b}^{(r)}(t)
$$

where

$$
\tilde{V}_{f}^{(r)}(t)=-\tilde{x}_{f}^{(r)^{\prime}}(t) \tilde{K}_{f}^{(r)}(t) \tilde{x}_{f}^{(r)}(t)+\tilde{q}_{f}^{(r)}(t)
$$

and

$$
\tilde{V}_{b}^{(r)}(t)=-\tilde{x}_{b}^{(r)^{\prime}}(t) \tilde{K}_{b}^{(r)}(t) \tilde{x}_{b}^{(r)}(t)+\tilde{q}_{b}^{(r)}(t) .
$$

Then the most probable distribution is $\tilde{\phi}^{\left(\tilde{r}_{s}(t)\right)}(\cdot)$ and the optimal smoother is given by

$$
\begin{aligned}
& \left(\tilde{r}_{s}(t), \tilde{x}_{s}(t)\right)=\left(\tilde{r}_{s}(t), \tilde{x}_{s}^{\left(\tilde{r}_{s}(t)\right)}(t)\right) \\
& =\left(\tilde{r}_{s}(t), \tilde{K}_{s}^{\left(\tilde{r}_{s}(t)\right)}(t)\left[\tilde{K}_{f}^{\left(\tilde{r}_{s}(t)\right)}(t) \tilde{x}_{f}^{\left(\tilde{r}_{s}(t)\right)}(t)\right.\right. \\
& \left.\left.+\tilde{K}_{b}^{\left(\tilde{r}_{s}(t)\right)}(t) \tilde{x}_{b}^{\left(\tilde{r}_{s}(t)\right)}(t)\right]\right)
\end{aligned}
$$

where $\tilde{K}_{s}^{\left(\tilde{r}_{s}(t)\right)}(t)=\left[\tilde{K}_{f}^{\left(\tilde{r}_{s}(t)\right)}(t)+K_{b}^{\left(\tilde{r}_{s}(t)\right)}(t)\right]^{-1}$.

The next theorem gives the near optimality of the limiting smoother.

Theorem 4.1 Let $\hat{x}_{s}^{(r), \epsilon}(t)$ and $\hat{x}_{s}^{\epsilon}(t)$ denote the smoothers defined by (14) and (15) respectively with $\phi^{(r)}(\cdot)$ replaced by $\phi^{(r), \epsilon}(\cdot)$. Then, for each $r \in \mathcal{N}_{0}$ and $t \in[0, T]$, we have

i) $\hat{x}_{s}^{(r), \epsilon}(t) \rightarrow \tilde{x}_{s}^{(r)}(t)$ as $\epsilon \rightarrow 0$.

ii) $\hat{x}_{s}^{\epsilon}(t) \rightarrow \tilde{x}_{s}(t)$ as $\epsilon \rightarrow 0$ if $\tilde{r}_{s}(t)$ is the unique minimizer of $\tilde{V}_{s}^{(r)}(t)$.

(Proof) See the appendix. (Q.E.D.)

\section{$5 \quad$ Numerical Examples}

In this section, we study numerical examples to demonstrate the effectiveness of the presented design algorithms.

We consider the following two mode systems and assume that the system parameters are as follows:

$$
\begin{gathered}
\dot{x}(t)=A(\theta(t)) x(t)+w(t, \theta(t)), \\
x(0)=x_{0}, \theta(0)=i_{0} \\
y(t)=H x(t)+v(t, \theta(t))
\end{gathered}
$$

where

- Mode 1:

$$
\begin{array}{r}
A_{1}=\left[\begin{array}{cc}
0 & 1 \\
-1 & -0.4
\end{array}\right], \quad A \\
H=[1,0]
\end{array}
$$

- Mode 2:

and

$$
M(t, i)=\left[\begin{array}{ll}
1 & 0 \\
0 & 1
\end{array}\right], N(t, i)=1, D_{0}=\left[\begin{array}{ll}
1 & 0 \\
0 & 1
\end{array}\right]
$$


for $i=1,2$. We set $\hat{x}_{0}=\operatorname{col}(-0.1,0)$ and the distribution of the initial mode $i_{0}$ as $(1 / 2,1 / 2)$. $w(\cdot)$ and $v(\cdot)$ are stochastic noises which aren't restricted to be Gaussian white. The candidates of mode distributions are given as follows:

$$
\phi^{(1)}=\left(\frac{2}{5}, \frac{3}{5}\right), \phi^{(2)}=\left(\frac{1}{10}, \frac{9}{10}\right), \phi^{(3)}=\left(\frac{7}{10}, \frac{3}{10}\right)
$$

Note that these distributions are limiting distributions if the mode transition law follows the Markovian jump processes. Actually the coreresponding Markovian transition matrices are

$$
\Pi^{(1)}=\left(\begin{array}{cc}
-3 & 3 \\
2 & -2
\end{array}\right), \Pi^{(2)}=\left(\begin{array}{cc}
-9 & 9 \\
1 & -1
\end{array}\right)
$$

and

$$
\Pi^{(3)}=\left(\begin{array}{cc}
-3 & 3 \\
7 & -7
\end{array}\right)
$$

respectively. The paths of $\theta(t)$ are generated randomly, and the performances are compared under the same circumstance, that is, the same set of the paths so that the performances can be easily compared.

We consider the whole system (23) with the true mode distribution $\phi^{(3)}$ over the time interval $t \in[0,15]$. We verify the effectiveness of the presented hybrid estimation algorithms and compare the estimation performances for the optimal filtering and smoothing algorithms. In order to carry out these algorithms we solve the forward or backward triplet of the differential equations (7)-(9) or (11)-(13) with the initial or terminal conditions for given observation $y(\cdot)$ and each candidate $r=1,2,3$ of given distributions, and obtain the pair $\left(\hat{r}(t), \hat{x}_{f}(t)\right)$ minimizing $V_{f}^{(r)}(t)$ in the filtering case or the pair $\left(\hat{r}_{s}(t), \hat{x}_{s}(t)\right)$ minimizing $V_{s}^{(r)}(t)$ in the smoothing case for $t \in[0, T]$. (If it is assumed that the mode transitions follow some Markovian jump processes, we consider that we solve the differential equations (17)-(19) or (20)-(22) and obtain the nearly optimal limiting estimators for $t \in[0, T]$.)

Filtered and smoothed values of the first components of the whole system states are given by Fig. $\mathbf{1}$ and Fig. 2 respectively. Fig. $\mathbf{3}$ and Fig. $\mathbf{4}$ show the square errors between the states and filtered values, and the states and smoothed values respectively. The mean square errors over the time interval $[0,15]$ are 0.0025 in the filtering case, and 0.0019 in the smoothing case respectively. From these figures and calculation results it is shown that the smoother gives better estimation than the filter. Filtered and smoothed mode distributions are given by Fig. 5 and Fig. 6. Notice that the vertical axes show the candidates of the mode distributions not the modes themselves. In Fig. 5 the filtered values of the mode distributions rapidly change to be left undecided. To the contrary in Fig. 6 the smoothed values of the mode distributions are firmly decided. Through these four figures it is shown that the

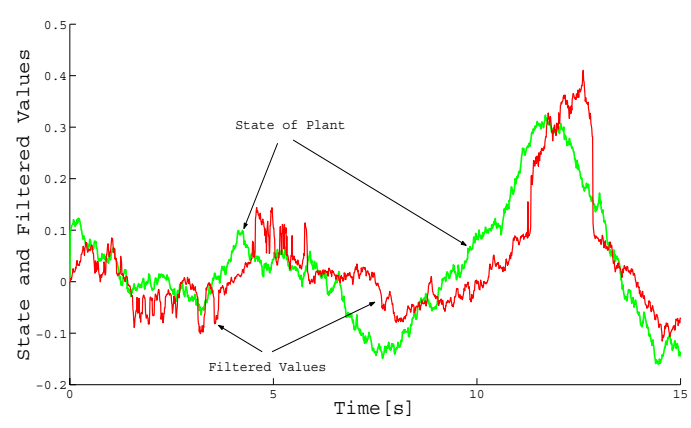

Fig. 1: The state of the system and filtered values

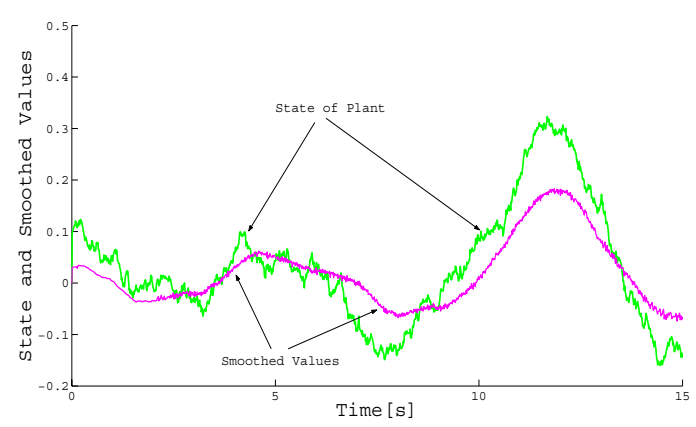

Fig. 2: The state of the system and smoothed values

optimal smoother presented in this paper gives better estimate perfromance than the optimal filter presented in the previous work ([23]) from the point of view of both state and modes estimation.

\section{Concluding Remarks}

In this paper we have studied the state and mode estimation problems for linear continuous-time hybrid systems over the fixed time interval. The systems aren't restricted to the Markovian jump systems except for Section 4 and the added noises aren't restricted to be Gaussian. We have adopted the MPT approach. The state and mode estimation approach adopted in this paper guarantees the optimality of estimation performance in the meaning of MPT different from the previous work $([5,9])$.

In this paper we have considered the problems that both the system state and modes are estimated. However we have considered the problems that the distributions of the modes over the fixed time interval not the modes themselves are estimated to grasp the global behavior of the hybrid systems over the long time intervals. In order to estimate both the system state and distributions of the modes we have introduced the averaged performance indices with respect to the candidates 


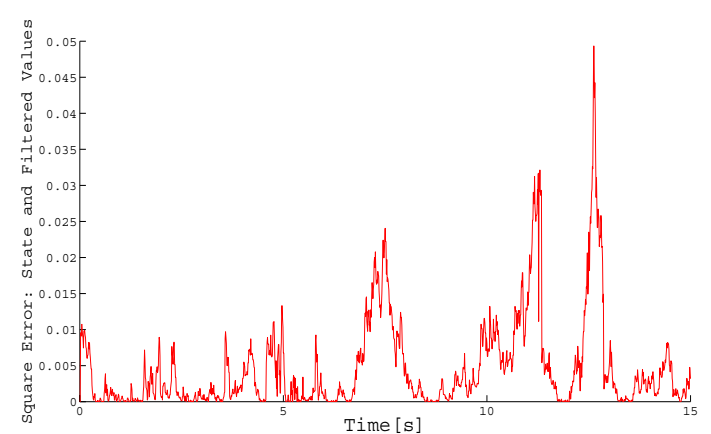

Fig. 3: The square errors between the state and filtered values

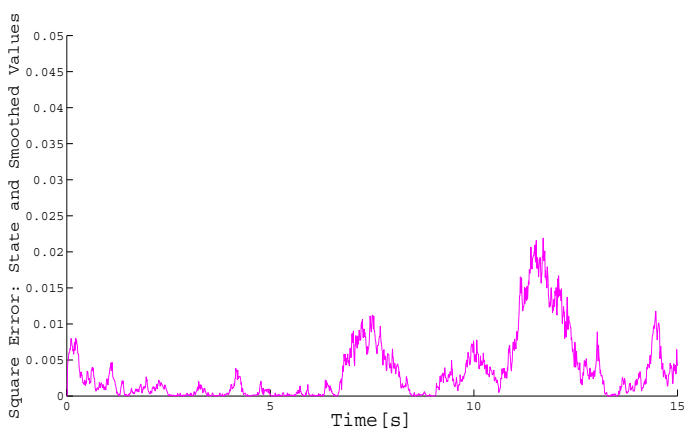

Fig. 4: The square errors between the state and smoothed values

of the mode distributions for the averaged systems. For these performance indices we have formulated the optimal filtering and smoothing problems for the observed information. The estimation problems have been reduced to the optimal control problems to find the noises minimizing the introduced performance indices. We have derived the forward and backward Riccati type differential equations and the forward and backward filters, which give the necessary conditions for the solvability of the optimal control problems. Then we have presented the optimal hybrid smoothing algorithm by the two filters approach. We have also considered the cases that the mode transitions follow the Markovian jump processes and shown the near optimality of the limiting estimators. Finally we have studied the numerical examples to compare the estimation performances by filtering and smoothing. We have obtained the better estimation performance by the smoothing algorithm than the filtering algorithm from the point of view of both state and modes estimation.

\section{References}

[1] T. Basar: Optimal Performance Levels for Minimax Filters, Predictiors and Smoothers, Syst. Contr. Lett.,

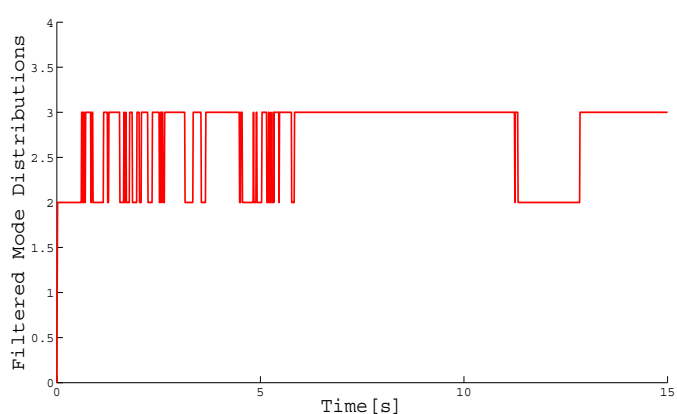

Fig. 5: The filtered mode distibutions

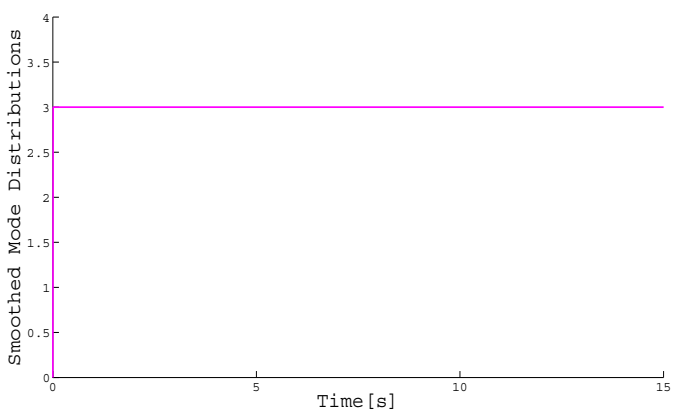

Fig. 6: The smoothed mode distributions

Vol. 16, Issue 5, pp. 309-317, 1991.

[2] E. Blanco, P. Neveux and G. Thomas: The $\mathrm{H}_{\infty}$ FixedInterval Smoothing Problem for Continuous Systems, IEEE Trans. Signal Process., Vol. 54, No. 11, pp. 40854090, 2006.

[3] H. A. P. Blom and Y. Bar-Shalom: Time-reversion of a hybrid state stochastic difference system with a jump-linear smoothing application, IEEE Trans. Inform. Theory, Vol. 36, No. 4, pp. 836-847, 1990.

[4] E. K. Boukas: Stochastic Switching Systems: Analysis and Design, Birkhauser, Boston, 2006.

[5] O. L. V. Costa: Linear Minimum Mean Square Error Estimation for Discrete-Time Markovian Jump Linear Systems, IEEE Trans. Automat. Contr., Vol. 39, No. 8, pp.1685-1689, 1994.

[6] O. L. V. Costa, M. D. Fragoso and R. P. Marques: Discrete-Time Markov Jump Linear Systems, Springer, London, 2005.

[7] H. Cox: Estimation of State Variables via Dynamic Programming, Proc. 1964 Joint Automatic Control Conf., Stanford, California, pp.376-381, 1964.

[8] V. Dragan and T. Morozan: The linear quadratic optimization problems for a class of linear stochastic systems with multiplicative white noise and Markovian jumping, IEEE Trans. Automat. Contr., Vol. 49, No. 5, pp. 665-675, 2004. 
[9] M. D. Fragoso, O. L. V. Costa, J. Baczynski and N. Rocha: Optimal linear mean square filter for continuous-time jump linear systems, IEEE Trans. Automat. Contr., Vol. 50, No. 9, pp.1364-1369, 2005.

[10] D. C. Fraser and J. E. Potter: The Optimum Linear Smoother as a Combination of Two Optimum Linear Filters, IEEE Trans. Automat. Contr., Vol. AC-14, No. 4, pp.387-390, 1969 .

[11] R. E. Helmick, W. D. Blair and S. A. Hoffman: FixedInterval Smoothing for Markovian Switching Systems, IEEE Trans. Inform. Theory, Vol. 41, No. 6, pp.1845$1855,1995$.

[12] A. H. Jazwinski: Filtering for Nonlinear Dynamical Systems, IEEE Trans. Automat., Contr., Vol. 11, pp.765-766, 1966.

[13] L. Ljung and T. Kailath: Backwards Markovian Models for Second-Order Stochastic Processes, IEEE Trans. Inform. Theory, Vol. 22, No. 4, pp.488-491, 1976.

[14] D. Q. Mayne: A Solution of the Smoothing Problem for Linear Dynamic Systems, Automatica, Vol. 4, No. 2, pp.73-92, 1966.

[15] J. S. Meditch: Orthogonal Projection and Discrete Optimal Linear Smoothing, J. SIAM Control, Vol. 5, No. 1, pp.74-89, 1967.

[16] G. Nakura: $\mathrm{H}_{\infty}$ Estimation for Linear ContinuousTime Markovian Jump Systems by Game Theoretic Approach, Proceedings of the 42nd ISCIE International Symposium on Stochastic Systems Theory and Its Applications (SSS10), Okayama, Japan, pp.154-162, 2010.

[17] H. E. Rauch: Solutions to the Linear Smoothing Problem, IEEE Trans., Automat., Contr., Vol. AC-8, pp.371-372, 1963.

[18] H. E. Rauch, F. Tung and C. T. Striebel: Maximum Likelihood Estimates of Linear Dynamic Systems, AIAA J., Vol. 3, No. 8, pp.1445-1450, 1965.

[19] G. S. Sidhu and U. B. Desai: New Smoothing Algorithms Based on Reversed-Time Lumped Models, IEEE Trans. Automat. Contr., Vol. 21, pp.538-541, 1976.

[20] D. D. Sworder and R. O. Rogers: An LQG Solution to a Control Problem with Solar Thermal Receiver, IEEE Trans. Automat. Contr., Vol. 28, pp.971-978, 1983.

[21] G. Verghese and T. Kailath: A Further Note on Backwards Markovian Models, IEEE Trans. Inf. Theory, Vol. IT-25, No. 1, pp.121-124, 1979.

[22] J. E. Wall, Jr., A. S. Willsky and N. R. Sandell, Jr.: On the Fixed-Interval Smoothing Problem, Stochastics, Vol. 5, pp.1-41, 1981

[23] Q. Zhang: Hybrid Filtering for Linear Systems with Non-Gaussian Disturbances, IEEE Trans. Automat. Contr., Vol. 45, pp.50-61, 2000.

\section{Appendix 1: Proof of Lemma 3.1}

In this appendix we show the positive-definiteness of the matrix $K_{b}^{(r)}(t)$.

(Proof of Lemma 3.1) For each $r \in \mathcal{N}_{0}$,

$$
\begin{aligned}
& V_{b}^{(r)}(t, x) \\
= & J_{b}^{(r)}\left(t, x, w_{b}^{(r) *}(\cdot)\right) \\
= & \int_{t}^{T} L^{(r)}\left(s, x^{*}(s), w_{b}^{(r) *}(s), y(s)\right) d s
\end{aligned}
$$

where $w_{b}^{(r) *}(s)=w_{b}^{(r) *}\left(s, x^{*}(s)\right)$ is the optimal control with

$$
\begin{aligned}
& w_{b}^{(r) *}(s, x) \\
=-\left(\bar{M}^{(r)}(s)\right)^{-1} & \left(K_{b}^{(r)}(s) x+p_{b}^{(r)}(s)\right. \\
& \left.+\bar{M}^{(r)}(s) \bar{A}^{(r)}(s) x-\overline{M A}^{(r)}(s) x\right)
\end{aligned}
$$

and

$$
\begin{gathered}
\dot{x}^{*}(s)=-\left(\bar{M}^{(r)}(s)\right)^{-1}\left(K_{b}^{(r)}(s)-\overline{M A}^{(r)}(s)\right) x^{*}(s) \\
-\left(\bar{M}^{(r)}(s)\right)^{-1} p_{b}^{(r)}(s), x^{*}(t)=x .
\end{gathered}
$$

Let $\Psi(s, t)$ be the state transition matrix of the linear time-varying system

$$
\dot{z}(s)=-\left(\bar{M}^{(r)}(s)\right)^{-1}\left(K_{b}^{(r)}(s)-\overline{M A}^{(r)}(s)\right) z(s)
$$

with $\Psi(t, t)=I$. Then we can show

$$
\frac{\partial x^{*}}{\partial x}=\Psi(s, t) \text { and } \frac{\partial^{2} x^{*}}{\partial x^{2}}=0 .
$$

Differentiating both sides of (24) twice with respect to $x$ and using the quadratic form of $V_{b}^{(r)}(t, x)$, we can obtain

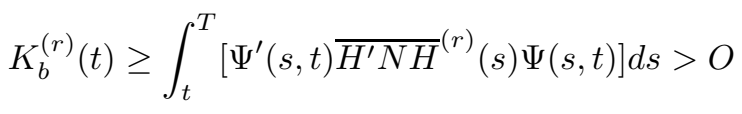

because of $M(t, i)>O, N(t, i) \geq O$ and $D>O$ and

$$
\begin{aligned}
& L^{(r)}\left(s, x^{*}(s), w_{b}^{*(r)}(s), y(s)\right) \\
:= & \sum_{i=1}^{m} \phi_{i}^{(r)}(s)\left(\left[\left(\bar{A}^{(r)}(s)-A(s, i)\right) x^{*}(s)+w_{b}^{*(r)}(s)\right]^{\prime}\right. \\
& \quad \times M(s, i)\left[\left(\bar{A}^{(r)}(s)-A(s, i)\right) x^{*}(s)+w_{b}^{*(r)}(s)\right] \\
+ & \left.\left(y(s)-H(s, i) x^{*}(s)\right)^{\prime} N(s, i)\left(y(s)-H(s, i) x^{*}(s)\right)\right)
\end{aligned}
$$

which concludes the proof. (Q.E.D.)

\section{Appendix 2: Proof of Proposition 4.1 and Theorem 4.1}

First the folllowing lemmas hold ([23]). 
Lemma 6.1 For each $r \in \mathcal{N}_{0}$ and $t \in[0, T]$, the inequalities

$$
\begin{gathered}
\left\|K_{f}^{(r)}(t)\right\| \leq C_{0}(T+1),\left\|p_{f}^{(r)}(t)\right\| \leq C_{0}(T+1), \\
\left\|q_{f}^{(r)}(t)\right\| \leq C_{0}(T+1) \\
\left\|K_{b}^{(r)}(t)\right\| \leq C_{T}(T+1),\left\|p_{b}^{(r)}(t)\right\| \leq C_{T}(T+1) \\
\text { and }\left\|q_{b}^{(r)}(t)\right\| \leq C_{T}(T+1)
\end{gathered}
$$

hold where $K_{f}^{(r)}(t), p_{f}^{(r)}(t), q_{f}^{(r)}(t), K_{b}^{(r)}(t), p_{b}^{(r)}(t)$ and $q_{b}^{(r)}(t)$ are the solutions of (7)-(9) and (11)-(13) respectively and

$$
\begin{gathered}
C_{0}=\max \left\{\operatorname { s u p } _ { t , i } \left\{\|M(t, i)\|\|A(t, i)\|^{2}\right.\right. \\
\left.+2\|N(t, i)\|\|H(t, i)\|^{2}\right\}, 2 \sup _{t, i}\left\{\|N(t, i)\|\|y(t)\|^{2}\right\}, \\
\left.2\left\|D_{0}\right\|, 2\left\|D_{0}\right\|\left\|\hat{x}_{0}\right\|^{2}\right\}, \\
C_{T}=\max \left\{\operatorname { s u p } _ { t , i } \left\{\|M(t, i)\|\|A(t, i)\|^{2}\right.\right. \\
\left.+2\|N(t, i)\|\|H(t, i)\|^{2}\right\}, 2 \sup _{t, i}\left\{\|N(t, i)\|\|y(t)\|^{2}\right\}, \\
\left.2\left\|D_{T}\right\|, 2\left\|D_{T}\right\|\left\|\hat{x}_{T}\right\|^{2}\right\} .
\end{gathered}
$$

Lemma 6.2 Let $F^{\epsilon}$ and $F$ be invertible matrices such that $F^{\epsilon} \rightarrow F$ as $\epsilon \rightarrow 0$. Then $\left(F^{\epsilon}\right)^{-1} \rightarrow F^{-1}$ holds.

\section{(Proof of Proposition 4.1)}

We only show the uniform convergence of $K_{b}^{(r), \epsilon}(\cdot)$. The others can be shown by the simimlar arguments.

By Lemma $6.1 K_{b}^{(r), \epsilon}(t)$ is uniformly bounded on $[0, T]$ for each $r \in \mathcal{N}_{0}$. Therefore, from (11), $\dot{K}_{b}^{(r), \epsilon}(t)$ is uniformly bounded on $[0, T]$ for each $r \in \mathcal{N}_{0}$, which implies the sequence $\left\{K_{b}^{(r), \epsilon}(\cdot)\right\}$ is equicontinuous on $[0, T]$. From Arzela-Ascoli theorem it follows that, for each sequence of $\{\epsilon \rightarrow 0\}$, there exists a subsecence (still indexed by $\epsilon$ ) such that $\left\{K_{b}^{(r), \epsilon}(t)\right\}$ uniformly converges to a continuous matrix-valued function $K^{(r), 0}(t)$. It suffices to show $K_{b}^{(r), 0}(t)=\tilde{K}_{b}^{(r)}$. In order to show this equality we write $K_{b}^{(r), \epsilon}(\cdot)$ as the integral form

$$
\begin{aligned}
K_{b}^{(r), \epsilon}(t) & \\
=D_{T}-\int_{t}^{T}\{ & K_{b}^{(r), \epsilon}(s)\left(\bar{M}^{(r), \epsilon}(s)\right)^{-1} K_{b}^{(r), \epsilon}(s) \\
& -{\overline{A^{\prime} M A}}^{(r), \epsilon}(s) \\
& {\overline{A^{\prime} M}}^{(r), \epsilon}(s)\left(\bar{M}^{(r), \epsilon}(s)\right)^{-1} \overline{M A}^{(r), \epsilon}(s) \\
- & -{\overline{H^{\prime} N H^{\prime}}}^{(r), \epsilon}(s) \\
& \left.\left.-K_{b}^{(r), \epsilon}(s)\left(\bar{M}^{(r), \epsilon}(s)\right)^{-1} K_{b}^{(r), \epsilon}(s)\right)^{-1} \overline{M A}^{(r), \epsilon}(s)\right\} d s
\end{aligned}
$$

where $\bar{F}^{(r), \epsilon}(t)=\sum_{i=1}^{m} \phi_{i}^{(r), \epsilon}(t) F(t, i)$ for any function $F(t, i)$.

The convergence $\phi_{i}^{(r), \epsilon}(t) \rightarrow \tilde{\phi}_{i}^{(r)}(t), \epsilon \rightarrow 0$ implies

$$
\begin{gathered}
\bar{M}^{(r), \epsilon}(t) \rightarrow \bar{M}_{\lambda}^{(r)}(t),{\overline{A^{\prime} M}}^{(r), \epsilon}(t) \rightarrow{\overline{A^{\prime} M_{\lambda}^{(r), \epsilon}}}^{(t),} \\
\overline{M A}^{(r), \epsilon}(t) \rightarrow \overline{M A}_{\lambda}^{(r)}(t)
\end{gathered}
$$

and

$$
{\overline{A^{\prime} M A}}^{(r), \epsilon}(t) \rightarrow{\overline{A^{\prime} M A_{\lambda}^{(r)}}}^{(t)} \text { as } \epsilon \rightarrow 0 .
$$

From Lemma 6.2 we have

$$
\left(\bar{M}^{(r), \epsilon}(t)\right)^{-1} \rightarrow\left(\bar{M}_{\lambda}^{(r)}(t)\right)^{-1} \text { as } \epsilon \rightarrow 0 .
$$

Letting $\epsilon \rightarrow 0$ and noting the uniform convergence $K_{b}^{(r), \epsilon}(t) \rightarrow K_{b}^{(r), 0}(t)$, we can show $K_{b}^{(r), 0}(t)$ corresponds to the integral form of the unique solution of (20). Therefore $K_{b}^{(r), 0}(t)=\tilde{K}_{b}^{(r)}(t)$. (Q.E.D.)

\section{( Proof of Theorem 4.1)}

By Proposition 4.1 and Lemma 6.2, we have

$$
\begin{aligned}
& \hat{x}_{f}^{(r), \epsilon}(t) \rightarrow \tilde{x}_{f}^{(r)}(t), \epsilon \rightarrow 0 \\
& \hat{x}_{b}^{(r), \epsilon}(t) \rightarrow \tilde{x}_{b}^{(r)}(t) \epsilon \rightarrow 0
\end{aligned}
$$

and

$$
\hat{x}_{s}^{(r), \epsilon}(t) \rightarrow \tilde{x}_{s}^{(r)}(t), \epsilon \rightarrow 0
$$

which imply $V_{f}^{(r), \epsilon}(t) \rightarrow \tilde{V}_{f}^{(r)}(t), V_{b}^{(r), \epsilon}(t) \rightarrow \tilde{V}_{b}^{(r)}(t)$ and $V_{s}^{(r), \epsilon}(t) \rightarrow \tilde{V}_{s}^{(r)}(t) \epsilon \rightarrow 0$. In order to show (ii) of Theorem 4.1 let denote $r_{0}$ denote the only minimizer of $\tilde{V}_{s}^{(r)}(t)$, i.e., $\tilde{V}_{s}^{\left(r_{0}\right)}(t)<\tilde{V}_{s}^{(r)}(t)$ for all $r \neq r_{0}$. Then $\tilde{r}_{s}(t)=r_{0}$ by definition. Thus, for sufficiently small $\epsilon>0$

$$
V_{s}^{\left(\tilde{r}_{s}(t)\right), \epsilon}(t)=V_{s}^{\left(r_{0}\right), \epsilon}(t)<V_{s}^{(r), \epsilon}(t) \text { for all } r \neq r_{0} .
$$

Hence let $\hat{r}_{s}^{\epsilon}(t)$ denote the only minimizer of $V_{s}^{(r), \epsilon}(t)$ and then $\hat{r}_{s}^{\epsilon}(t)=\hat{r}_{s}(t)$ holds for sufficiently small $\epsilon>0$. Therefore

$$
\begin{aligned}
& \left|\hat{x}_{s}^{\epsilon}(t)-\tilde{x}_{s}(t)\right|=\left|\sum_{r=1}^{n_{0}} \mathbf{1}_{\left\{\tilde{r}_{s}(t)=r\right\}}\left(\hat{x}_{s}^{(r), \epsilon}(t)-\tilde{x}_{s}^{(r)}(t)\right)\right| \\
& \quad \leq \sum_{r=1}^{n_{0}} \mathbf{1}_{\left\{\tilde{r}_{s}(t)=r\right\}}\left|\hat{x}_{s}^{(r), \epsilon}(t)-\tilde{x}_{s}^{(r)}(t)\right|
\end{aligned}
$$

holds as $\epsilon \rightarrow 0$ where the indecator function $\mathbf{1}_{\left\{\tilde{r}_{s}(t)=r\right\}}:=$ 1 if $\tilde{r}_{s}(t)=r$, and $\mathbf{1}_{\left\{\tilde{r}_{s}(t)=r\right\}}:=0$ if $\tilde{r}_{s}(t) \neq r$. (Q.E.D.) 